\title{
CONSTRUÇÃO DE UMA FONTE DE CORRENTE E DE UMA SONDA PARA MEDIDA DE CONDUTIVIDADE PELO MÉTODO DA SONDA DE QUATRO PONTAS
}

\author{
Olacir Alves Araújo \\ Unidade Universitária de Ciências Exatas e Tecnológicas, Universidade Estadual de Goiás, Br 153, km 98, 75000-000 \\ Anápolis - GO \\ Wilson Botter Júnior* \\ Instituto de Química, Universidade Federal de Goiás, Campus Samambaia, CP 131, 74001-970 Goiânia - GO \\ Jesiel Freitas Carvalho e Ediron Lima Verde \\ Instituto de Física, Universidade Federal de Goiás, Campus Samambaia, CP 131, 74001-970 Goiânia - GO
}

Recebido em 13/2/02; aceito em 26/3/03

\begin{abstract}
THE CONSTRUCTION OF AN ELETRICAL CURRENTE SOURCE AND OF A PROBE FOR CONDUCTIVITY MEASUREMENT BY A FOUR POINT PROBE METHOD. This paper describes the construction of an eletrical current source and of a probe to be used in the measurement of eletrical conductivity through a four-point probe method. These pieces of equipments can be obtained at the low price of US\$50.00 and are adequate for eletrical conductivity measurements in the semiconductor range, that is from $10^{-1}$ to $10^{-6} \mathrm{~S} \mathrm{~cm}^{-1}$.
\end{abstract}

Keywords: four-point probe method; conductivity; conducting polymer.

\section{INTRODUÇÃO}

A condutividade elétrica de filmes, lâminas e de superfícies condutoras é um parâmetro físico essencial quando se deseja utilizar materiais em aplicações elétricas e eletrônicas ${ }^{1}$. O método mais extensivamente utilizado para esse propósito é o sistema de quatro pontas $^{2,3}$, que consiste em medir os valores de corrente e voltagem, em eletrodos independentes, a partir dos quais se pode obter a resistividade em função da geometria da amostra. $\mathrm{O}$ sistema, originalmente descrito na literatura, é constituído por um sensor formado por quatro eletrodos verticais, cujas pontas estão em um mesmo plano, orientadas colinearmente e regularmente espaçadas. Dois eletrodos estão acoplados a uma fonte de corrente e os outros dois a um voltímetro ${ }^{4}$. Usualmente, os eletrodos externos servem como injetores e coletores de corrente, enquanto a diferença de potencial gerada é medida nos eletrodos internos. Entretanto, é possível a utilização de outras disposições geométricas, bem como a injeção de corrente através dos eletrodos internos ${ }^{1,4}$. A medida é realizada, quando o sensor entra em contato com a superfície da amostra. Utilizam-se molas controladoras da pressão, para garantir a qualidade desse contato entre as quatro pontas e a amostra ${ }^{5}$. Esse método minimiza os erros de medida, devido à baixa resistência de contato entre os eletrodos e a amostra ${ }^{6,7}$, além de ser útil na caracterização de propriedades elétricas de superfícies condutoras, tais como a mudança de condutividade devido à oxidação superficial, como descrito por Petersen ${ }^{8}$.

Sistemas de quatro pontas para medidas de condutividade elétrica podem ser adquiridos comercialmente. Entretanto, em muitas situações pode ser conveniente, ou mesmo necessário, construir o próprio equipamento. O propósito deste trabalho foi desenvolver um sistema de quatro pontas, associando simplicidade e baixo custo, para medida de condutividade elétrica em amostras semicondutoras depositadas sobre superfícies isolantes, com resultados equivalentes aos obtidos em equipamentos comerciais, na faixa da semicondução.

\section{O MÉTODO DA SONDA DE QUATRO PONTAS}

O método da sonda de quatro pontas baseia-se na aplicação de uma corrente constante entre dois eletrodos e na medida da tensão entre os outros dois. A diferença de potencial entre as pontas pode ser avaliada supondo que, em lugar das pontas de corrente, existam fontes pontuais de carga $+\mathrm{Q}$ e $-\mathrm{Q}{ }^{9}$. Pode-se assumir, em alguns casos, que as pontas metálicas possuem dimensões infinitesimais e que as dimensões das amostras são semi-infinitas ${ }^{10}$. Em amostras volumosas, nas quais a espessura $w$ da amostra é muito maior que o espaçamento $s$ entre os eletrodos $(w>s)$, assume-se que há uma protuberância esférica de corrente emanando das pontas dos eletrodos externos cuja área é $\mathrm{A}=2 \pi x^{2}$, correspondente a uma superfície hemisférica ${ }^{10}$. Em camadas muito finas $(w<s)$, assume-se que há emanação circular de corrente ao invés de esférica; nesse caso a área será $\mathrm{A}=2 \pi x w$ e a resistência diferencial será dada por:

$d R=\rho\left(\frac{d x}{A}\right)$

onde: $\mathrm{R}$ é a resistência, $\rho$ é a resistividade, $\mathrm{dx}$ é o elemento diferencial de comprimento e A é a seção de área pela qual flui a corrente $\boldsymbol{I}$.

Integrando-se sobre o comprimento entre os dois eletrodos internos e, considerando-se que a diferença de potencial entre esses é induzida por cada um dos eletrodos externos, pois ambos são fontes de correntes de mesma intensidade mas direções opostas, haverá sobreposição das correntes e, conseqüentemente, $\mathrm{R}=\mathrm{V} / 2 \boldsymbol{I}^{10-12}$. Assim, obtêm-se as Equações (2) e (3) para amostras volumosas $(w>>$ s) e finas $(w<<s)$, respectivamente.

$\rho=2 \pi s\left(\frac{\mathrm{V}}{\mathrm{I}}\right)$

$\rho=\frac{\pi w}{\ln 2}\left(\frac{\mathrm{V}}{l}\right)$ 
Em geral, a Equação 3 pode ser expressa como:

$\rho=C\left(\frac{V}{l}\right) w$

aqui C é um fator proveniente da integração geométrica. Seu valor dependerá das dimensões da amostra, de sua forma geométrica, do espaçamento entre os eletrodos e da amostra estar depositada sobre substrato isolante ou condutor. Considerando-se amostras semi-infinitas, retangulares e depositadas sobre superfícies isolantes, C será igual a 4,5324; ou seja, o valor de $\pi / \ln 2$. Os valores de $C$ para amostras retangulares e circulares, depositadas sobre superfícies isolantes ou condutoras foram determinados por Smits ${ }^{6}$, Valdes ${ }^{11}$ e Uhlir ${ }^{12}$. Descrições mais detalhadas da utilização do método da sonda de quatro pontas estão disponíveis na literatura ${ }^{13}$.

\section{PARTE EXPERIMENTAL}

A Figura 1 mostra a configuração utilizada para construir o sistema de medida de condutividade elétrica, com destaque para a sonda de quatro pontas. Foram utilizados eletrodos de latão, de $5,0 \mathrm{~cm}$ de comprimento por 2,0 $\mathrm{mm}$ de diâmetro, de cabos de multímetro adquiridos comercialmente. Esses eletrodos foram decapados, por 3-4 min, em solução de ácido nítrico, $0,1 \mathrm{~mol} \mathrm{~L}^{-1}$. As pontas foram, então, cuidadosamente afiadas e moldadas em ácido nítrico concentrado. Utilizou-se o espaçador de teflon (Figura 1) para limitar o comprimento das pontas, cujo diâmetro, obtido em função do tempo de permanência no ácido, é de $1 \mathrm{~mm}$. O revestimento com ouro foi realizado por eletrodeposição, utilizando-se como ânodo um fio de ouro em solução de $\mathrm{KCN} / \mathrm{AuCN}$, com concentrações, respectivamente, de $0,010 \mathrm{~mol} \mathrm{~L}^{-1}$ e $0,015 \mathrm{~mol} \mathrm{~L}^{-1}$ e densidade de corrente de 0,2-0,3 $\mathrm{A} \mathrm{dm}^{-2}$. Opcionalmente, o revestimento pode ser realizado por um ourives. Foram feitos quatro furos colineares de $2 \mathrm{~mm}$ de diâmetro em uma peça cilíndrica de teflon com $5 \mathrm{~cm}$ de diâmetro e $1 \mathrm{~cm}$ de espessura. $\mathrm{O}$ espaçamento entre os furos foi de $4 \mathrm{~mm}$ a partir de cada centro geométrico. Essa distância dependerá do diâmetro dos eletrodos e da precisão do serviço mecânico utilizado. A diminuição no espaçamento entre os eletrodos é recomendada, pois quanto menor a distância entre esses, melhores serão os resultados experimentais, uma vez que se minimizam os erros provocados por

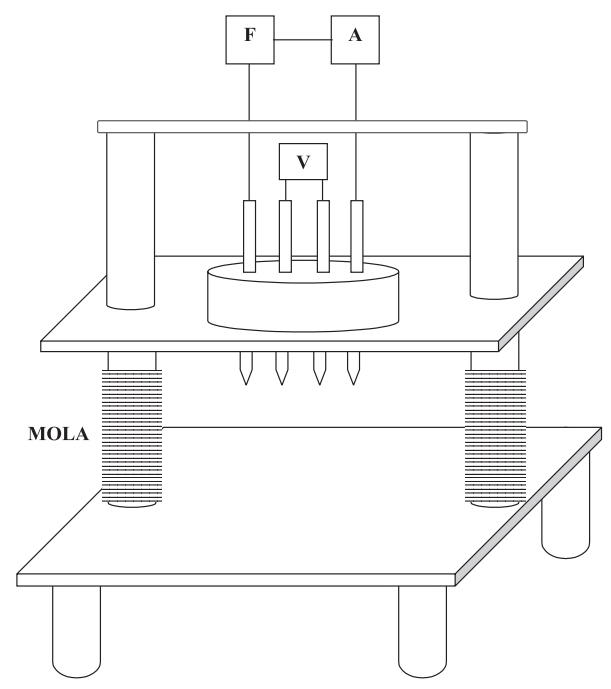

Figura 1. Esquema mostrando a configuração utilizada no sistema quatro pontas: $(F)$ fonte de corrente; $(A)$ amperímetro $e(V)$ voltímetro variações na espessura da amostra. A peça cilíndrica de teflon serviu como cabeça do suporte dos eletrodos, a qual foi fixada em um porta-amostras com base de teflon. Entre esse porta-amostras e a cabeça do suporte dos eletrodos colocaram-se duas molas para controlar a pressão exercida sobre a amostra.

O gerador de corrente (Figura 2) é constituído por uma fonte de tensão bipolar $( \pm 18 \mathrm{~V})$ necessária para alimentação simétrica dos operacionais envolvidos no fornecimento da corrente pré-ajustada. O circuito integrado A1 possui uma tensão de referência estável, que é utilizada para conversão em corrente de saída. O operacional A2 faz com que a corrente em R1 seja Vref / Rc, entretanto, para correntes constantes maiores, o que implica uma Rc menor, é necessário um gerador de corrente de maior potência. O conjunto A2 e Tr formam, juntos, um estágio de potência com a carga aterrada via coletor de Tr. Essa configuração permite estabilidade de corrente da ordem de $20 \mathrm{ppm} /{ }^{\circ} \mathrm{C}$, na faixa de $500 \mathrm{~mA}$, desde que o valor de R3 seja no mínimo de $33 \Omega$. O conjunto todo deve ser ligado a um terra local e a caixa do circuito deve ser de alumínio ou outro metal, para uma boa blindagem contra ruídos externos. Os operacionais A1 e A2 são do tipo Ca 3140, e a fonte de tensão de referência é constituída pelo operacional LM723. O potenciômetro multivoltas P2 é responsável pelo ajuste da corrente na carga e os resistores R1 e R2 são filmes metálicos com resistência de $1 \mathrm{~K} \Omega$ e $1 / 4 \mathrm{~W}$ de dissipação máxima.

Os eletrodos externos da sonda de quatro pontas foram conectados à fonte de corrente e a um eletrômetro analógico Keithley 602, funcionando como amperímetro; os eletrodos internos, conectados a um voltímetro digital ET-2000 Minipa. As medidas de condutividade foram realizadas em filmes de polipirrol sintetizados sobre membranas de silicona, que foram cortados com dimensões de $2 \times 2 \mathrm{~cm}$. Para cada amostra foram feitas três medidas, em cada lado, variando-se o potencial de saída da fonte; considerou-se a média dos seis valores como o resultado final.

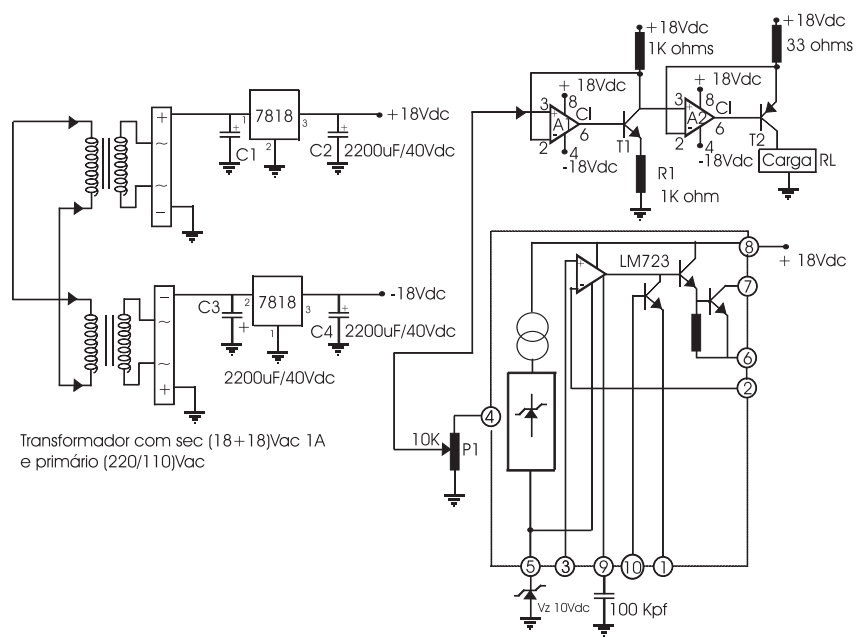

Figura 2. Esquema da fonte de corrente construída para uso no sistema de quatro pontas

\section{RESULTADOS E DISCUSSÃO}

A fonte de corrente, cuja construção descrevemos neste trabalho, mostrou-se bastante estável na faixa de corrente medida, que foi de $5,0 \times 10^{-9}$ A a $3,0 \times 10^{-6} \mathrm{~A}$. Seu custo, desconsiderando-se os medidores e indicadores de corrente, foi de, aproximadamente, R\$ 100,00 (US\$ 30,00). O suporte e a sonda de quatro pontas foram conseguidos por, aproximadamente, R\$ 65,00 (US\$ 20,00) não inclusos os serviços de confecção das peças, que foram realizadas nas 
oficinas da UFG. A comparação entre esses valores e aqueles apresentados pelas sondas comerciais ${ }^{13}$ denota a vantagem situacional da construção de um equipamento.

Os resultados de condutividade elétrica obtidos com o condutivímetro, foram comparados aos resultados obtidos utilizando-se um sistema de quatro pontas adquirido comercialmente, que chamamos condutivímetro de referência, na Tabela 1. Os valores mostrados correspondem à média das seis medidas realizadas para cada amostra, sendo três em cada superfície. Esses resultados possuem a mesma ordem de grandeza, sendo possível medir condutividades elétricas de até $10^{-6} \mathrm{~S} \mathrm{~cm}^{-1}$, ou seja, na faixa da semicondução. Não foi possível medir condutividades inferiores a $10^{-6} \mathrm{~S} \mathrm{~cm}^{-1}$, mas seria possível melhorar a sensibilidade do sistema, alterando alguns componentes eletrônicos da fonte de corrente e utilizando um voltímetro mais sensível. As flutuações entre os valores das medidas do condutivímetro e do sistema de referência, entretanto, não são representativas do ponto de vista do significado das medidas. O condutivímetro mostrou-se perfeitamente adequado para medidas de condutividade elétrica na faixa da semicondução, embora não seja indicado para medidas de condutividade de bons condutores e isolantes. A construção do equipamento mostrou-se simples e economicamente viável, possibilitando-se a utilização da técnica em situações onde o equipamento comercial não esteja disponível.

Tabela 1. Valores de condutividade elétrica obtidos para filmes de polipirrol sintetizados sobre borracha de silicona, utilizando o método da sonda de quatro pontas

\begin{tabular}{ccc}
\hline Amostra & $\begin{array}{c}\text { condutivímetro } \\
\sigma\left(\mathrm{S} \mathrm{cm}^{-1}\right)\end{array}$ & $\begin{array}{c}\text { condutivímetro de } \\
\text { referência* } \sigma\left(\mathrm{S} \mathrm{cm}^{-1}\right)\end{array}$ \\
\hline 1 & $5,2 \times 10^{-2}$ & $4,2 \times 10^{-2}$ \\
2 & $2,3 \times 10^{-2}$ & $5,6 \times 10^{-2}$ \\
3 & $7,1 \times 10^{-3}$ & $4,8 \times 10^{-3}$ \\
4 & $5,6 \times 10^{-6}$ & $4,7 \times 10^{-6}$ \\
\hline
\end{tabular}

* Uma fonte de corrente programável 224-Keithley, um eletrômetro programável 617-Keithley e um sensor de quatro pontas com espaçamento de $2 \mathrm{~mm}$.

\section{CONCLUSÕES}

A construção de uma fonte de corrente e de uma sonda para a medida de condutividade elétrica pelo método da sonda de quatro pontas é possível a um custo aproximado de US\$ 50,00. Esses equipamentos mostraram-se adequados para medidas de condutividade elétrica de até $10^{-6} \mathrm{~S} \mathrm{~cm}^{-1}$. A construção e utilização dos equipamentos descritos neste trabalho podem ser úteis em situações em que o equipamento comercial não esteja disponível para utilizações rotineiras.

\section{AGRADECIMENTOS}

Ao Prof. Dr. A. E. Job, da Faculdade de Ciência e Tecnologia da UNESP de Presidente Prudente-SP, pelas medidas de condutividade elétrica no sistema de sonda de quatro pontas utilizado como referência.

\section{REFERÊNCIAS}

1. Zrudsky, D. R.; Bush, H. D.; Fassett, J. R.; Rev. Sci. Instrum. 1966, 37, 885 .

2. Kennedy, J. K.; Rev. Sci. Instrum. 1962, 33, 773.

3. Petersen, C. L.; Hansen, T. M.; Boggild, P.; Boisen, A.; Hansen, O.; Grey, F.; Sens. Actuators, A 2001, 3153.

4. Skotheim, T. A.; Handbook of Conducting Polymers, Ed. Marcel Dekker: New York, 1986.

5. Paulnack, C. L.; Chaplin, N. J.; Rev. Sci. Instrum. 1962, 33, 873.

6. Smits, F. M.; Bell Sys. Tech. J. 1958, 37, 711.

7. Keywell, F.; Dorosheski, G.; Rev. Sci. Instrum. 1960, 31, 833.

8. Petersen, C. L.; Grey, F.; Aono, M.; Surf. Sci. 1997, 676, 377.

9. Baranauskas, V.; Técnicas instrumentais de caracterização de semicondutores, Ed. da UNICAMP: Campinas, 1989.

10. http://argon.eecs.berkeley.edu:8080/ ee143/Four-Point_Probe/, acessada em Outubro 2000.

11. Valdes, L. B.; Proc. Inst. Rad. Eng. 1954, 42, 420.

12. Uhlir Jr., A.; Bell Sys. Tech. J. 1955, 34, 105.

13. Girotto, E. M.; Santos, I. A.; Quim. Nova 2002, 25, 639. 